\title{
CORRESPONDENCE
}

\section{Reply to "Comments on 'A Wave-Resolving Simulation of Langmuir Circulations with a Nonhydrostatic Free-Surface Model: Comparison with Craik-Leibovich Theory and an Alternative Eulerian View of the Driving Mechanism""}

\author{
YASUSHI FUJIWARA ${ }^{\mathrm{a}}$ AND YUTAKA YOSHIKAWA \\ Graduate School of Science, Kyoto University, Kyoto, Japan \\ YOSHIMASA MATSUMURA \\ Atmosphere and Ocean Research Institute, The University of Tokyo, Tokyo, Japan
}

(Manuscript received 20 January 2019, in final form 5 February 2019)

\begin{abstract}
Fujiwara et al. explicitly simulated Langmuir circulations using a wave-resolving simulation (WRS) technique and found that the residual wave effect on vorticity was well represented by the vortex force of the Craik-Leibovich (CL) equation, at least in the simulated situation. In response to the simulation results, Mellor has proposed a view that ubiquitous applicability of the CL formulation is still questionable and that the three-dimensional radiation stress (3DRS) formulation that he has derived encompasses both of the vortex force effect and an effect that is lower order in terms of wave steepness. Here, these opinions are discussed in terms of the approximations used in the wave-averaged formulations. The asymptotic expansion of the Eulerian-averaged momentum equation allows the separate discussion of two different wave effects: pressure correction and torque. It is argued that the approximation adopted in Mellor's 3DRS formulation is presumably not accurate enough to properly parameterize the wave torque effect, and possible approaches to examine its performance are proposed. We agree with the view that the applicability of the CL formulation needs further investigation. WRS will be a helpful tool for this purpose.
\end{abstract}

\section{Introduction}

Using a latest nonhydrostatic free-surface numerical model, Fujiwara et al. (2018, hereinafter FYM) performed a wave-resolving simulation (WRS) to study the driving mechanism of simulated Langmuir circulations. In their analysis, FYM found that the simulated wave-induced torque is very well represented by the curl of the vortex force in the Craik-Leibovich (CL) equation (Craik and Leibovich 1976, hereinafter CL76) in that particular case.

Mellor (2019, hereinafter M19) recently submitted a comment on FYM. M19 reviews the numerical work of FYM and puts forward his view on the various

\footnotetext{
${ }^{\text {a }}$ Research Fellow, Japan Society for the Promotion of Science, Tokyo, Japan.
}

Corresponding author: Yasushi Fujiwara, fujiwara@kugi.kyotou.ac.jp wave-averaged formulations such as the three-dimensional radiation stress (3DRS) equation (Mellor 2003, 2015, hereinafter M03 and M15, respectively) and the CL equation. M19 shares the common question with FYM: when does the CL equation properly parameterize the residual wave effect, and when does it not? His answer is as follows: (i) its ubiquitous applicability is still questionable, and (ii) the 3DRS of M15 contains both the vortex force and an effect that is lower order in terms of wave steepness and is missing in CL76.

In this reply, we discuss the opinions of M19 through the consideration of the wave effect terms in the waveaveraged momentum equation. Since wave-averaged formulations essentially approximate the wave-associated fluctuation to obtain a useful parameterization of its residual effects, it is crucial to know the required accuracy of the approximation. Here we feature the Eulerian wave-averaged momentum equation to identify two different wave effects (pressure correction and torque) 
and the accuracy of the wave-associated fluctuation required to approximate these effects. This separation of effects clarifies the "lower-order effect" mentioned by M19.

\section{Required accuracy of wave motion in the Eulerian framework}

Many wave-averaged formulations are derived using the asymptotic expansion (e.g., Longuet-Higgins and Stewart 1962; Craik and Leibovich 1976; McWilliams et al. 2004; Aiki and Greatbatch 2014). While it might not be so physically intuitive as nonasymptotic derivations (e.g., Longuet-Higgins and Stewart 1964; Smith 2006), the asymptotic expansion allows us a solid discussion of accuracy and errors with the hierarchical series of equations. Here, we shall discuss the required accuracy of approximations based on the asymptotic approach, partly following the derivation by CL76.

In the Eulerian wave-averaged momentum equation, the residual wave effect is

$$
-\nabla\left(\frac{1}{2} \overline{\left|\mathbf{u}^{\prime}\right|^{2}}\right)+\overline{\mathbf{u}^{\prime} \times \boldsymbol{\omega}^{\prime}} .
$$

Here $\mathbf{u}$ is velocity and $\boldsymbol{\omega}$ is vorticity. The overline and prime denote the Eulerian temporal average over one (or several) wave period(s) and the deviation from the Eulerian temporal average, respectively. The first term is a gradient of a scalar and does not affect vorticity. It does modify the pressure field, whose horizontal variation changes mean surface elevation, the effect known as the wave setup/setdown. The second term is responsible for any forces accompanied by torque. The expression in Lamb's form [Eq. (1)] is useful to distinguish different wave effects, namely, pressure correction and torque.

The use of different coordinate systems leads to different forms of wave-averaged equations, but they should essentially contain the same physics behind. Therefore, the wave-averaged equation based on the Eulerian average [Eq. (1)] and the one based on the $s$ coordinate (M03, M15) are equivalently accurate as long as we have the completely accurate field of the wave-associated fluctuation (quantities such as $\mathbf{u}^{\prime}$ ). In practice, we usually do not have such information because there is no general analytic solution of waves in the presence of background current and topography. One possible solution is to explicitly simulate wave motions (i.e., WRS) and thereby numerically obtain all the information of both wave-averaged motion and fluctuation, but it is computationally demanding and thus often unreasonable for realistic simulations. Another approach is to use an asymptotically approximate form of $\mathbf{u}^{\prime}$, etc. to obtain useful parameterizations of the wave effect terms.

In the following paragraphs, we shall discuss the accuracy of the approximated wave orbital motions required to properly parameterize the wave residual effect terms in the Eulerian-averaged form [Eq. (1)]. We feature the Eulerian framework because it provides a clear view of the terms responsible for the pressure correction and torque effects, which act with different magnitudes. To facilitate the discussion, we follow the scaling assumptions adopted by CL76, namely, (i) the wave-averaged velocity $\overline{\mathbf{u}}$ is $O\left(\varepsilon^{2} c\right)$, and (ii) the spatial scale of $\overline{\mathbf{u}}$ is $O\left(k^{-1}\right)$, where $\varepsilon$ is wave steepness, $c$ is wave phase speed, and $k$ is wavenumber. Under these assumptions, the wave-averaged flow $\overline{\mathbf{u}}$ and the fluctuation (wave motion in the leading order) from the averaged flow $\mathbf{u}^{\prime}$ are expanded in series:

$$
\begin{aligned}
& \mathbf{u}^{\prime}=\varepsilon \mathbf{u}_{1}^{\prime}+\varepsilon^{2} \mathbf{u}_{2}^{\prime}+\varepsilon^{3} \mathbf{u}_{3}^{\prime}+\cdots, \quad \text { and } \\
& \overline{\mathbf{u}}=\varepsilon^{2} \overline{\mathbf{u}}_{2}+\varepsilon^{3} \overline{\mathbf{u}}_{3}+\cdots .
\end{aligned}
$$

The first- and the second-order fluctuation $\mathbf{u}_{1}^{\prime}$ and $\mathbf{u}_{2}^{\prime}$ follow the equations

$$
\begin{aligned}
& \partial \mathbf{u}_{1}^{\prime} / \partial t=-\nabla p_{1}, \quad \text { and } \\
& \partial \mathbf{u}_{2}^{\prime} / \partial t=-\nabla\left(p_{2}+\left|\mathbf{u}_{1}^{\prime}\right|^{2} / 2\right),
\end{aligned}
$$

where $p$ denotes the kinematic pressure minus the hydrostatic pressure from a fixed level $\left(P / \rho_{0}+g z\right.$ using the raw pressure $P$ and the reference density $\rho_{0}$ ). Compressibility, viscosity, stratification, and system rotation are neglected, and $\partial \overline{\mathbf{u}} / \partial t \ll \partial \mathbf{u}^{\prime} / \partial t$ is used assuming the temporal scale separation. Because only gradient forces are present in the rhs of Eqs. (4) and (5), the fluctuation is irrotational up to the second order $\left(\boldsymbol{\omega}_{1}^{\prime}=\boldsymbol{\omega}_{2}^{\prime}=0\right)$. This justifies the use of the Airy wave (the irrotational linear wave) solution to approximate $\mathbf{u}_{1}^{\prime}$. The third-order fluctuation $\mathbf{u}_{3}^{\prime}$ follows

$$
\partial \mathbf{u}_{3}^{\prime} / \partial t=-\nabla\left(p_{3}+\mathbf{u}_{1}^{\prime} \cdot \mathbf{u}_{2}\right)+\mathbf{u}_{1}^{\prime} \times \overline{\boldsymbol{\omega}}_{2} .
$$

Therefore, $\mathbf{u}_{3}^{\prime}$ reflects the interaction of the background current $\overline{\mathbf{u}}$ and the leading-order wave motion $\mathbf{u}_{1}^{\prime}$, and it is accompanied by vorticity $\left(\boldsymbol{\omega}_{3}^{\prime} \neq 0\right)$ due to the nongradient term $\mathbf{u}_{1}^{\prime} \times \overline{\boldsymbol{\omega}}_{2}$.

Now let us consider which order of wave-associated fluctuation $\mathbf{u}^{\prime}$ contributes to the wave torque, which is 
caused by the term $\overline{\mathbf{u}^{\prime} \times \boldsymbol{\omega}^{\prime}}$. Substituting the series expansion (2) into the term $\overline{\overline{\mathbf{u}}^{\prime} \times \boldsymbol{\omega}^{\prime}}$ using the fact $\boldsymbol{\omega}_{1}^{\prime}=\boldsymbol{\omega}_{2}^{\prime}=0$, we obtain

$$
\overline{\mathbf{u}^{\prime} \times \boldsymbol{\omega}^{\prime}}=\varepsilon^{4}\left(\overline{\mathbf{u}_{1}^{\prime} \times \boldsymbol{\omega}_{3}^{\prime}}\right)+(\text { higher-order terms }) .
$$

Therefore, the parameterization of the leading-order torque effect requires the information of the third-order fluctuation (fluctuating vorticity; see also Fig. 7 of FYM). In other words, the Airy wave solution (the first-order wave) alone is unable to represent the torque effect in the Eulerian derivation. CL76 dealt with the wave-averaged vorticity equation and showed that $\nabla \times\left(\overline{\varepsilon \mathbf{u}_{1}^{\prime} \times \varepsilon^{3} \boldsymbol{\omega}_{3}^{\prime}}\right)$ can be transformed to $\nabla \times\left(\mathbf{u}^{\mathrm{St}} \times \varepsilon^{2} \overline{\boldsymbol{\omega}}_{2}\right)$, where $\mathbf{u}^{\mathrm{St}}$ is the Stokes drift and is $O\left(\varepsilon^{2} c\right)$. After "uncurling" the waveaveraged vorticity equation, we obtain the wave-averaged momentum equation with the vortex force and an unknown gradient force (Leibovich 1977). ${ }^{1}$

Next, let us consider the pressure correction effect. Substituting the series expansion (2) into the term $-\nabla\left(\overline{\left|\mathbf{u}^{\prime}\right|^{2}} / 2\right)$ yields

$$
-\nabla\left(\frac{1}{2} \overline{\left|\mathbf{u}^{\prime}\right|^{2}}\right)=-\varepsilon^{2} \nabla\left(\frac{1}{2} \overline{\left|\mathbf{u}_{1}^{\prime}\right|^{2}}\right)+(\text { higher-order terms }),
$$

where the leading-order term $-\varepsilon^{2} \nabla\left(\overline{\left|\mathbf{u}_{1}^{\prime}\right|^{2}} / 2\right)$ is unaffected by the wave-averaged current $\overline{\mathbf{u}}$. Therefore, the information of $\mathbf{u}_{1}^{\prime}$ (i.e., the Airy wave) would be sufficiently accurate to capture the setup/setdown. ${ }^{2}$

To summarize, the asymptotic expansion of the Eulerian-averaged momentum equation clarifies the two different wave effects, namely, the pressure correction and torque, and the accuracy required to properly parameterize these effects. The Airy wave solution would be sufficiently accurate to parameterize the pressure correction, while the higher-order solution with the wave-current interaction effect must be used to parameterize the leading-order torque. The pressure correction is "the lower-order effect" mentioned by M19, and it cannot be parameterized through the "uncurling" of wave-averaged vorticity equation, as was done in

\footnotetext{
${ }^{1}$ Mellor (2016) and M19 argue that there is some missing term in the CL equation that was dropped in "curl-uncurl" processes. Recall that such missing information must take a gradient form.

${ }^{2}$ This argument is somewhat crude because the term $\overline{\mathbf{u}^{\prime} \times \boldsymbol{\omega}^{\prime}}$, whose divergence is generally nonzero, can also cause wave setup/ setdown. When the horizontal variation of $\overline{\left|\mathbf{u}^{\prime}\right|^{2}} / 2$ is small, the wave setup/setdown effect from $-\nabla\left(\left|\overline{\mathbf{u}}^{\prime}\right|^{2} / 2\right)$ can be smaller than from $\overline{\mathbf{u}^{\prime} \times \boldsymbol{\omega}^{\prime}}$. In such cases, the higher-order fluctuation $\mathbf{u}_{3}^{\prime}$ must be taken into account to correctly estimate the setup/setdown.
}

CL76 and Leibovich (1977). Meanwhile, as noted before, the pressure correction does not affect vorticity.

\section{Discussion and conclusions}

M03 and M15 derived a wave-averaged momentum equation using the so-called $s$ coordinate, which follows the fluctuating upper surface. The wave motion in the residual wave effect terms was approximated with the Airy wave solution in the derivations [similar to truncating the second- and higher-order terms in Eq. (2)], obtaining the second-order accurate 3DRS $\left(F_{\alpha}\right.$ in M19). This approximation is less accurate than the one required to derive the torque effect in the Eulerian formulation, that is, the interaction of the wave-averaged current and the lower-order wave motion that appears in the higher-order fluctuation is neglected.

Although the Eulerian discussion cannot be directly applied to the $s$ coordinate, we consider that the torque effect parameterization of M03/M15 needs to be carefully examined. As suggested by the opinion ii of M19, the horizontal component of the M03/M15 formulation does contain a vortex force term due to horizontal Stokes drift, which arises from expanding the advection term of the total momentum ( $U_{\alpha}$ in M19). However, the 3DRS term $F_{\alpha}$ is second-order accurate and may contain an error that contaminates the leading-order torque effect terms, which is typically higher-order than $F_{\alpha}$ (e.g., the fourthorder in CL76). Also, while unmentioned in M19, the vertical component of the wave-averaged momentum equation needs to be discussed.

As the reliability of the torque effect parameterization in the Airy wave-based formulation is poorly supported, we suggest that solid experimental or theoretical evidence should be given. One simple way to give such supports would be to compare the vorticity evolution in a simulation based on the Airy wavebased formulation and a corresponding WRS. A reference result of a simple test (e.g., the development of laminar Langmuir circulations under monochromatic waves in a periodic domain) with a well-controlled WRS will be useful, and the authors are currently preparing such a result. Another more theoretical way might be to derive a wave-averaged vorticity equation using the asymptotic expansion and examine the leading-order wave torque term from the general 3DRS (i.e., including all higher-order motions). If it is properly represented with Airy wave approximation or less significant than the torque terms corresponding to the vortex force term in Eq. (12) of M19, the Airy wave approximation would be sufficiently accurate. The asymptotic discussion in the present manuscript could be 
used as a guideline for such discussion. These attempts will clarify whether the accuracy of the approximation is sufficient or not, and either conclusion will be largely beneficial for the users of the formulations.

Based on various assumptions and frameworks, various wave-averaged formulations have been proposed. Some formulations include both the wave setup/ setdown effect and the torque effect (e.g., McWilliams et al. 2004; Smith 2006; Ardhuin et al. 2008). However, it is always worthwhile to pay attention to the validity of the formulations because the assumptions that they are based on may not be strictly satisfied all over the simulated domain and period, ${ }^{3}$ where overlooked terms in the derivation may become significant. Also, the models of wave momentum evolution become questionable when there is small-scale current due to turbulence or fronts because they are at best based on the wave action conservation and the ray equation (e.g., McWilliams et al. 2004), which are satisfied only when the spatial scale of the current is much larger than the wavelength. It is important to monitor the performance of formulations in such cases. In this sense, the opinion $\mathrm{i}$ of M19 (ubiquitous application of the CL equation is questionable) is important.

Again, well-controlled WRS will be a useful tool to examine the performance of wave-averaged formulations. Using WRS, FYM gave a support for the vortex force parameterization of the torque effect in the simulated particular conditions, which roughly satisfied the asymptotic assumptions of CL76. ${ }^{4}$ As mentioned in their conclusion, there are many other possible situations yet to be studied, and further investigation is required to expand our understanding of wave-current interaction in the upper ocean. When WRS is used in such attempts, careful examinations of numerical model performance and analysis method are necessary since the residual wave effect on current is quite subtle compared to the forces driving orbital motions.

Acknowledgments. This research is supported by Grant-in-Aid for Scientific Research on Innovative Areas (MEXT KAKENHI Grants JP15H05824 and JP15H05825) and Grant-in-Aid for JSPS Research Fellow (JSPS KAKENHI Grant JP17J07923).

\footnotetext{
${ }^{3}$ For example, in turbulent flows the energy spans over a wide range of spatiotemporal scales, and there is likely a scale at which the asymptotic assumption (e.g., $\overline{\mathbf{u}}$ varies over distance $k^{-1}$ ) is not satisfied.

${ }^{4}$ With the parameters listed at the end of the section 3 of FYM, $|\overline{\mathbf{u}}| \sim 0.1 \mathrm{~m} \mathrm{~s}^{-1}, \varepsilon^{2} c \sim 0.01 \mathrm{~m} \mathrm{~s}^{-1}$, and (the spatial scale of $\left.\overline{\mathbf{u}}\right) \sim k^{-1}$. The conditions of CL76 (see section 2) are roughly satisfied with an error of one order of magnitude.
}

\section{REFERENCES}

Aiki, H., and R. J. Greatbatch, 2014: A new expression for the form stress term in the vertically Lagrangian mean framework for the effect of surface waves on the upper ocean circulation. J. Phys. Oceanogr., 44, 3-23, https://doi.org/10.1175/JPO-D12-0228.1.

Ardhuin, F., N. Rascle, and K. A. Belibassakis, 2008: Explicit waveaveraged primitive equations using a generalized Lagrangian mean. Ocean Modell., 20, 35-60, https://doi.org/10.1016/ j.ocemod.2007.07.001.

Craik, A. D. D., and S. Leibovich, 1976: A rational model for Langmuir circulations. J. Fluid Mech., 73, 401-426, https:// doi.org/10.1017/S0022112076001420.

Fujiwara, Y., Y. Yoshikawa, and Y. Matsumura, 2018: A wave-resolving simulation of Langmuir circulations with a nonhydrostatic freesurface model: Comparison with Craik-Leibovich theory and an alternative Eulerian view of the driving mechanism. J. Phys. Oceanogr., 48, 1691-1708, https://doi.org/10.1175/ JPO-D-17-0199.1.

Leibovich, S., 1977: Convective instability of stably stratified water in the ocean. J. Fluid Mech., 82, 561-581, https://doi.org/ 10.1017/S0022112077000846.

Longuet-Higgins, M. S., and R. Stewart, 1962: Radiation stress and mass transport in gravity waves, with application to 'surf beats.' J. Fluid Mech., 13, 481-504, https://doi.org/ 10.1017/S0022112062000877.

— and - 1964: Radiation stresses in water waves; A physical discussion, with applications. Deep-Sea Res. Oceanogr. Abstr., 11, 529-562, https://doi.org/10.1016/0011-7471(64)90001-4.

McWilliams, J. C., J. M. Restrepo, and E. M. Lane, 2004: An asymptotic theory for the interaction of waves and currents in coastal waters. J. Fluid Mech., 511, 135-178, https://doi.org/ 10.1017/S0022112004009358.

Mellor, G. L., 2003: The three-dimensional current and surface wave equations. J. Phys. Oceanogr., 33, 1978-1989, https:// doi.org/10.1175/1520-0485(2003)033<1978:TTCASW > 2.0. $\mathrm{CO} ; 2$.

- 2015: A combined derivation of the integrated and vertically resolved, coupled wave-current equations. J. Phys. Oceanogr., 45, 1453-1463, https://doi.org/10.1175/JPO-D-140112.1.

- 2016: On theories dealing with the interaction of surface waves and ocean circulation. J. Geophys. Res. Oceans, 121 , 4474-4486, https://doi.org/10.1002/2016JC011768.

_ 2019: Comments on "A wave-resolving simulation of Langmuir circulations with a nonhydrostatic free-surface model: Comparison with Craik-Leibovich Theory and an alternative Eulerian view of the driving mechanism." J. Phys. Oceanogr., 49, 885-888, https://doi.org/10.1175/JPO-D-18-0222.1.

Smith, J. A., 2006: Wave-current interactions in finite depth. J. Phys. Oceanogr., 36, 1403-1419, https://doi.org/10.1175/ JPO2911.1. 